\title{
Construction and Reform of Modern Digital Signal Processing Course in Background of Electric Power
}

\author{
SUN Zheng \\ Department of Electronic and Communication Engineering \\ North China Electric Power University \\ Baoding, P.R.China \\ sunzheng_tju@163.com
}

\begin{abstract}
This paperexplores the teaching reform of Modern Digital Signal Processing course for graduate students majored in electric power engineering and information \& communication engineering in electric power university. Based on the features of this curriculum, the reform was implemented through optimizing the course content, following the development of top-ranking textbooks, adopting various instructional patterns, designing rich practices and using flexible assessment method. The reform achieved desired results in our past teaching practice. The students greatly improved their learning interest and practical ability in comparison to previous students. These results demonstrated that the teaching reform conforms to the development trend of the specialty and the requirements for qualified talent training.
\end{abstract}

Keywords—modern digital signal processing; teaching reform; electric power background; major of electronics; information and communication

\section{INTRODUCTION}

Digital signal processing (DSP) technologies originated between the 17th and 18th century. Currently, it has become an important modernized tool of the scientific research. It mainly studies methods for expressing signals with numbers or symbolic sequences and processing these sequences with numerical techniques. The purpose is to filter noise and interference, to estimate characteristic parameters of signals, and to transform signals into other forms, etc. Besides classical numerical analysis methods, an amazing breakthrough in theory and application has occurred. A large number of complex and precise algorithms emerged with the rapid development of high-speed computers and microprocessors as well as the overlapping and integration of different disciplines. The formation of modern DSP theory and technology was marked by modern spectral analysis and estimation, modern filtering and intelligent information processing. It has been applied in almost all engineering fields. Especially, the application of digital signal processing technology in the power system is becoming more and more extensive since the construction of smart grid was put forward.

At present, Modern Digital Signal Processing (MDSP) course is mainly for graduate students in Chinese engineering colleges and universities. It is the theoretical basis for the specialty of communication, electronic and information. As a connecting link in the teaching process, it lays the foundation for other professional knowledge. Also, it is the improvement in the field of signal processing after the study of undergraduate course of Signal and System and Digital Signal Processing Foundation. It aims to improve the theoretical analysis ability and the ability for students to solve practical engineering problems. It lays the foundation for scientific research on power system communication, fault detection of electrical equipment, smart grid and other related fields. It also provides important basic theory and analytical tool for researchers and engineering technicians engaged in the fields of communication and information processing.

MDSP curriculum involves more complex and deeper theory in comparison to the undergraduate course of Digital Signal Processing Foundation. The research objects are discrete random signals rather than deterministic discrete signals. The signal processing system is nonlinear time-varying system, such as adaptive filter, and multiple-input-multiple-output system rather than linear time-invariant system and singleinput-single-output system. The signal transform concerns about a joint analysis of time and frequency $[1,2]$. Therefore, the requirement for the mathematical basis of students is stricter and higher in the study of this course. Students need to comprehensively apply linear algebra, complex function, matrix theory, numerical analysis, probability theory and mathematical statistics to solve the problems of signal processing and system analysis in engineering practice. As a course combining theory and practice, the involved theories and methods have been widely applied in various fields. Thus, the course content is generally based on the current development of information science and technology to enable students to contact with advanced technologies [3].

MDSP is a required degree course for master of engineering majored in Information and Communications Engineering and Electronic and Communication Engineering of North China Electric Power University. The main objectives of the two specialties in the electric power background are to train excellent masters specialized in electronic information and communication with distinctive features of energy and electricity. That is, the goal is to cultivate professional talents with expertise and technology in electronics, information, network, and communication. Therefore, students should not only master the systematic knowledge in their own specialty, but also have creative thinking to discover, analyze and solve practical engineering problems. Besides, they should transfer or 
apply the knowledge to explore unknown scientific and engineering field.

In the teaching process, we adopted several methods to construct the curriculum including constructing teacher team, optimizing course content, following the development of topclass teaching materials, combining various teaching methods, designing various practices and using flexible assessment methods. These methods are based on the curriculum features and the development trend of electronic, information and communication in the power electric background. Our goal is to help students to master related theories and technologies and to improve the learning efficiency and practice ability.

\section{CONSTRUCTING TEACHER TEAM WITH ENGINEERING BACKGROUND}

We built a teacher team with strong teaching, research and innovation ability and good comprehensive quality. The team focuses on foundation, strengthens practice and emphasizes quality cultivation. The teachers' ages, professional titles and educational backgrounds are of rational structure. The team consists of 6 young and middle-aged front-line teachers with an average age of 40 years old. It includes 2 professors, 2 associate professors, 1 lecturers and 1 engineers with a educational structure of 5 doctors and 1 masters. Teachers not only have many years of experience in teaching work, but also have strong background of theoretical research and engineering application. They also have stable research direction including modern sensing and signal detection, intelligent detection of power equipment, machine learning, computer vision, optical fiber sensing and DSP system design, etc. They have made outstanding achievements in their respective research fields. They can lead students to some advanced theories and technologies of digital signal processing in the field of power electronics and communications.

\section{OPTIMIZING COURSE CONTENT}

We considered the lack of curriculum hours, e.g., about 32 hours in our school, and the difficulty of the content. Then, we carefully selected and integrated main theoretical contents into six modules according to the training requirements of electronic information and communication specialty in the power background.

\section{A. Review of Digital Signal Processing Basics}

This module includes basic concepts of discrete-time signals and systems, time-domain and frequency-domain analysis of discrete time signal and system, Z-domain analysis of discrete time signal and system and design of digital filters, etc.

\section{B. Modern Signal Analysis Basics}

This module includes time-frequency conjoint analysis of signals, multi-resolution analysis of signals, time-duration and frequency bandwidth of signals, instantaneous frequency of signals, signal decomposition, basic concept of Frame, Poisson summation formula, and Zak transform, etc.

\section{Multirate Signal Processing}

This module includes concept of multirate, decimation and interpolation of discrete signals, multirate signal processing based on signal decimation and interpolation, multirate signal processing implemented with filter bank and applications of multirate signal processing, etc.

\section{Stationary Random Signal Analysis}

This module includes concept and characteristic description of random signals, concept of stationary random signals, timedomain statistical characteristics of stationary random signals, ergodic property of stationary random signals, and analysis of a linear system excited by a stationary random signal, etc.

\section{E. Power Spectrum Density (PSD) Estimation}

This module includes concept and characteristics of power spectrum, quality evaluation of PSD, classical PSD methods including periodogram method, smoothing periodogram method and autocorrelation (AR) method, quality evaluation of classical PSD methods, influence factors on the quality of PSD, improvement of PSD methods, parametric models of stationary random signals, typical modern PSD methods including linear prediction AR model method and maximum entropy spectral estimation (MESE) method, recursive Levinson-Durbin algorithm, lattice predictor, Burg recursive method, least squares method based on forward and backward linear prediction, comparison of the performance of modern PSD methods and applications of PSD in signal detection, etc.

\section{F. Adaptive Signal Processing}

This module includes principle of adaptive filtering, adaptive linear combiner, mean square error analysis, adaptive least mean square (LMS) algorithm, adaptive recursive least squares RLS algorithm, LMS algorithm implementation based on IIR adaptive filter with recursive structure, digital realization of adaptive filters, calculation examples for adaptive filters, least square adaptive filter and its structure of lattice filter, fast transversal filter (FTF) adaptive algorithm, and applications of adaptive signal processing, etc.

\section{SELECTING PROPER TEACHING MATERIAL}

It is important to choose a suitable textbook for classroom teaching by teachers and self-study by students. The teaching material needs to meet the requirements of the development of the subject and the teaching reform. It should not only cover the course content we have preferred, but also have a clear logic and reasonable arrangement of the chapters. It should not only include a complete theoretical system, but also provide typical and representative examples, especially the problems of programming practice.

We finally chose Digital Signal Processing Theory, Algorithm and Implementation (Third Edition) compiled by $\mathrm{Hu}$ Guangshu of Tsinghua University. This book is rich in content, including classical digital signal processing and statistical digital signal processing, and also includes some frontiers. The content arrangement focuses on the combination of theory and application. It pays special attention to the application of Matlab to implement the theories and algorithms, and also 
more consideration on how to help teaching and reader's selflearning [4]. The book consists of two parts. Part I involves digital signal processing basics including analysis of discrete signal and system, Z transform (ZT), discrete Fourier transform (DFT), fast Fourier transform (FFT), design of infinite impulse response (IIR) and finite impulse response (FIR) filter, finite word-length problem and hardware implementation of digital signal processing, etc. Part II discusses statistical digital signal processing including description of random signal, stationary random signal, autocorrelation function estimation, classical PSD estimation, modern PSD estimation, Wiener filtering and adaptive filtering, etc. The book also briefly introduces functions provided in Matlab. In the last section of each chapter (except for the $10^{\text {th }}$ chapter and the $11^{\text {th }}$ chapter), the Matlab files involved in this chapter are given. The reader can learn how to use Matlab to realize signal processing.

\section{COMBINING VARIOUS TEACHING METHODS}

We scientifically and efficiently utilized various teaching methods based on advanced teaching concepts and psychological characteristics of students in accordance with the curriculum requirements. These methods stimulated the students' interest in learning, research and innovation and improved the initiative and effectiveness of the study.

\section{A. Theory Teaching in Classroom}

In the classroom teaching, we used a combination of three complementary methods including blackboard, multimedia and discussion by considering the curriculum features such as many abstract contents, fast update and few class hours, etc. Consequently, the efficiency of teacher-teaching and studentlearning was improved.

The blackboard teaching was used to derive and prove relatively simple and strict logic and to explain simple examples, such as the formula of time-duration and frequency bandwidth of a signal, the convolution-correlation theorem, the concept of PSD and the Levinson-Durbin recursive formula, etc. In this way, students had a relatively sufficient time for thinking and reviewing the knowledge they have learned in the past.

Multimedia teaching was used to intuitively display the material for expanding knowledge and to improve the teaching efficiency by means of visual thinking. We included three types of materials: a) directly displayed materials, such as complex formulas, waveform of signals, tables and case results, etc; b) presentation of unimportant contents, such as the development history of random signal processing and the research progress of PSD estimation, etc; c) expanding knowledge, such as new technologies in this field and case analysis of speech signal processing and image processing.

Class discussion is centered on students and is based on the idea of heuristic teaching. We mainly used it as the supplementation to other teaching activities. Students could freely select the subjects according to their own interests. They prepared research reports and PowerPoint (PPT) presentation before class. They could focus on hot topics or specific applications of DSP in engineering practice. The teacher chose better ones from them in advance. Then, the selected students introduced the research or investigation results through PPT in class. After the presentation, the teacher and other students put forward some questions and everybody expressed his own view. The teacher guided students to actively participate in the learning activities improving their interest.

The three teaching methods are complementary to each other effectively improving the teaching efficiency and solving the problem of less curriculum hours and more content. At the same time, the teacher shared the courseware, exercises with answers, and Matlab source codes of typical examples with students in the teaching process. It was convenient for students to review the contents after class and to expand their thinking for scientific research work.

\section{B. Case Presentation}

The teachers with rich scientific research experience in the course team gave special lectures and displayed specific cases related to digital signal processing. Case representation included five topics: power equipment monitoring and intelligent defect detection, application of wavelet analysis in power system, application of computer vision in smart grid, fault detection of submarine cable based on optical fiber sensing and biomedical electromagnetic imaging and image processing. The teacher guided students to be in close contact with the research progress and frontiers in the field of power electronics and communication.

\section{Network Teaching}

We developed a network course using multimedia and network technology. Also, we often supplemented and updated the website. The remote network teaching and guidance enhanced the role of communication and interaction between teachers and students. With the help of network teaching platform, students could learn independently, flexibly control the progress and content and repeat learning some knowledge points so as to improve their learning effect.

\section{DESIGNING VARIOUS PRACTICES}

We designed comprehensive, self-designed and open experiments with existing experimental conditions to improve students' practical ability to analyze and solve problems independently, to innovate and to express themselves. The topics mainly included realization of signal processing algorithm and system through Matlab programming and DSP hardware. Objects being processed were different kinds of signals, such as voice, image, human physiological signals, e.g., electrocardiograph (ECG) and electroencephalograph (EEG), and remote sensing signals, etc. The problems to be solved involved filtering, feature extraction, PSD estimation, and image recognition, etc. Through the training, students could skillfully use Matlab and DSP techniques to analyze signals and design and simulate signal processing systems. This is conducive to strengthen the understanding of basic theories and concepts. Also, it is a necessary ability for further study and research in the future. 


\section{REFORMING ASSESSMENT METHOD}

The previous method to evaluate the students' learning effect of MDSP course was only final open-book examination. In general, test questions were to assess students' ability to apply theoretical knowledge to solve comprehensive calculation problems and to prove the principle and formula. In order to pass the examination, students usually recited boring theories and obscure formulas by rote in a short time. In this way, the teachers could not objectively evaluate the students' ability to solve specific problems in the engineering practice with theoretical knowledge. The professionals we cultivate should have professional theoretical knowledge and innovation and practical ability according to the development trend of science and technology and requirements for talents majored in electronics, information and communication in the background of big power. Therefore, we reformed the assessment method by taking into account the evaluation of the application ability. The score of the final open-book examination accounted for only $50 \%$ of the total. The class discussion and practice were brought into the assessment. Students were required to write research reports for practical topics and make PPT reports on the seminar. Teachers gave reasonable scores so as to evaluate the learning effectiveness scientifically.

\section{CONCLUSION}

The specialty of electronics, information and communication in the background of electric power has the characteristics of combining information and communication technology with the development of energy. The trained talents should have a series of important abilities. For example, they should master professional knowledge of modern communication, signal and information processing system. They should research new theory and technology to solve technical problems independently. They should be able to apply broadband communication, electronic information, Internet of things and cloud computing technologies to the power system industry so as to provide efficient, reliable and safe information transmission for the development of smart grid.
As a compulsory degree course for master graduate students majored in the specialty of electronics, communication and information engineering, the teaching mode and content of MDSP course must follow the development of the specialty in the new period and the requirements for professional talents. Practice has proved that the traditional mode of teaching in classroom is difficult to cultivate students' learning habits and ability. The author has taught graduate students majored in electronic and communication engineering and information and communication engineering in North China Electric Power University (Baoding) adopting the new teaching mode described in this paper for three years (2016 2018). The teaching process with distinctive features showed that the reform has achieved the desired results. The students' learning interest, practical ability and total achievement have been greatly improved compared with previous students. Students generally reflected that the study of MDSP was no longer boring in this way. They learned real knowledge and skills for the future research work. Also, they had a clear and thorough understanding of the current progress and future prospects of their specialties.

\section{ACKNOWLEDGMENT}

This work was supported by Project of High Quality Curriculum Construction for Graduate Students of North China Electric Power University.

\section{REFERENCES}

[1] Wang Qiusheng, Yuan Haiwen, Cui Yong, “Teaching probe and practice to Modern Digital Signal Processing,” Journal of Beijing University of Aeronautics and Astronautics (Social Sciences Edition), vol. 22, pp.7780, April 2009.

[2] Li Mei, Liu Junhua, "Teaching reform and practice of course of Modern Digital Signal Processing,” Chinese Geological Education, vol.3, pp.8587, 2010.

[3] Gao Yuan, Yuan Haiying, Wu Yan, "Teaching reform and practice of Modern Digital Signal Processing Course,” Education Teaching Forum, vol.16, pp.93-94, 2012.

[4] Hu Guangshu, Digital Signal Processing Theory, Algorithm and Implementation (Third Edition). Tsinghua University Press, 2012, pp.10-15. 